\title{
Spectral near field data of LED systems for optical simulations
}

Ingo Rotscholl, Klaus Trampert, Udo Krüger, Franz Schmidt

Ingo Rotscholl, Klaus Trampert, Udo Krüger, Franz Schmidt, "Spectral near field data of LED systems for optical simulations," Proc. SPIE 11144, Photonics and Education in Measurement Science 2019, 111440P (17 September 2019); doi: 10.1117/12.2531258

SPIE Event: Joint TC1 - TC2 International Symposium on Photonics and Education in Measurement Science 2019, 2019, Jena, Germany 


\title{
Spectral near field data of LED systems for optical simulations**
}

\author{
Ingo Rotscholl*a, Klaus Trampert ${ }^{\mathrm{b}}$, Udo Krüger ${ }^{\mathrm{a}}$, Franz Schmidt ${ }^{\mathrm{a}}$ \\ ${ }^{a}$ TechnoTeam Bildverarbeitung GmbH, Werner-von-Siemens-Str. 5, 98693, Ilmenau, Germany \\ ${ }^{b}$ Light Technology Institute, Karlsruhe Institute of Technology, Engesserstr. 13, 76131, Karlsruhe, \\ Germany
}

\begin{abstract}
This work presents, summarizes and validates a fast, accurate and general measurement and modeling technique to obtain spectral near field data of LED systems in order to improve the optical design process of modern high quality LED systems. It requires only a minimum of goniophotometric near field measurements as well as no time-consuming angularly resolved spectral measurements. The procedure is named physically motivated basis spectra (PMBS) as its main assumption is that each piece of angularly and spatially varying spectral information can be described as the weighted sum of its physical basis spectra such as the individual semiconductors or a phosphor. Based on detailed spectral information regarding the goniophotometric measurement setup, the spectral model is obtained by solving a simple system of linear equations using the obtained near field measurements. The complete process is validated and applied to different state-of-the-art LED systems. The obtained results can be used directly in state-of-the-art ray tracers.

**Please note that this conference paper is a summary which consists solely of selected extracts, from the freely available Ref. [1]. (This paper is completely published under CC BY-SA 4.0 from [1])
\end{abstract}

Keywords: Photometry, Radiometry, Light-emitting diodes, Ray tracing, Illumination design, Optical engineering

\section{INTRODUCTION}

Light-emitting diodes (LEDs) have become one of the most important light sources in lighting technology. LED lighting applications range from medical [2,3], horticulture [4] and automotive lighting [5] over several general lighting applications such as street lighting, work place lighting as well as shop and home lighting [6]. The development of these systems often requires the optical designer to include the near field radiation properties of each light source in the system [7,8]. By considering the complete light source information in state-of-the-art Monte Carlo ray tracers, a high "lighting quality" can be ensured. In modern lighting technology, the term "lighting quality" refers to a permanently increasing number of different aspects, which also depends on the specific application. Besides photometric quantities and efficiency this may include visual and aesthetic properties such as uniformity, color uniformity and color rendering or non-visual effects of light on human beings [9-11]. Examples of undesired non-uniformities are shown in Fig 1. All these aspects depend on the spectrum of the light source. In contrast to conventional incandescent or fluorescent lamps, modern LED multi-chip systems as well as plain white LEDs can show strong spectral variations in both the angular and the spatial dimension, which can in general not be measured directly with conventional near field goniophotometers.
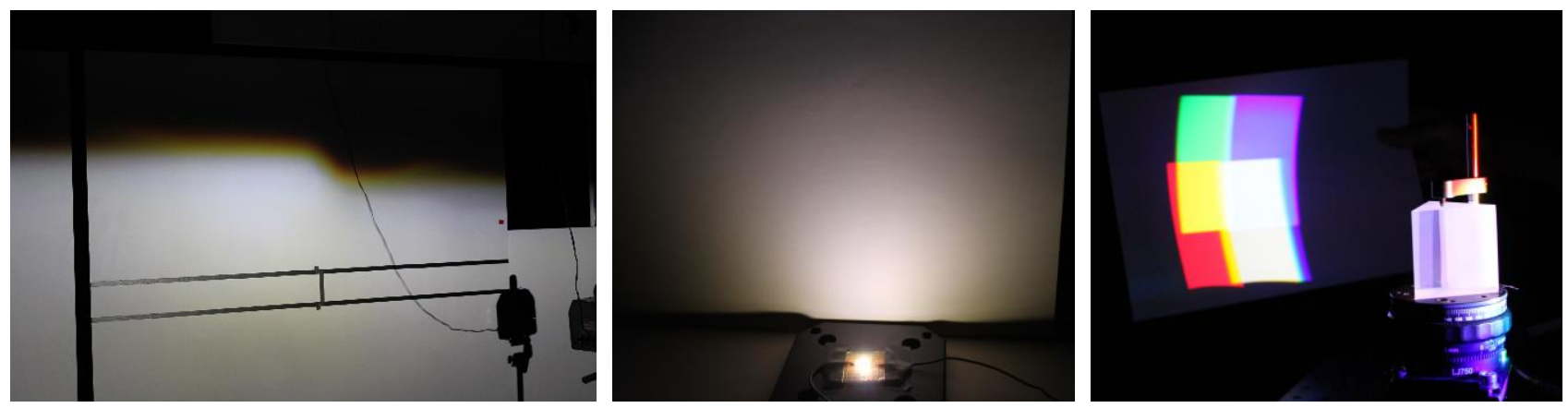

Figure 1: Examples of spectrally varying sources and optical system which lead color non-uniformities (published under CC BY-SA 4.0 from [1]). 
Recent methods to model or measure spectral ray data were proposed during the last years [12-18]. Most of them combine conventional ILMD-based (imaging luminance measurement device) near field goniophotometry with different optical filters and spectral measurements [12-16]. While most of them are capable of generating directly useable data, they may either only describe angular variations as [15], are limited to specific LEDs by principle as [16] or require a large amount of measurement or modeling time as [12-15, 17, 18]. Especially a high number of angular resolved spectral measurements increases the measurement time because the integration time of a spectrometer is higher than that of an ILMD.

The aim of this contribution is to present and verify a fast method to generate spectral ray data, which can be applied to all LED systems. The next section describes the mathematical concept and the general procedure. Afterwards aspects regarding the practical implementation are briefly discussed. More details regarding each topic is available in the cited literature or [1] in general.

\section{PHYSICALLY MOTIVATED BASIS SPECTRA}

The plenoptic function $R(X, Y, Z, \varphi, \vartheta, \lambda)$ describes the radiation of a source in terms of starting point $(X, Y, Z)$ direction $(\vartheta, \lambda)$ and wavelength $\lambda$ if temporal variations and polarization can be neglected. It is therefore the most complete description of a radiation source if the ray optic assumption is used [19]. However, each directly measured ray file $M(X, Y, Z, \varphi, \vartheta)$ represents only the 5-dimensional non-spectral plenoptic function because the spectral information is lost due to the optical integration on the ILMD.

\section{General concept}

The main idea of the Physically Motivated Basis Spectra (PMBS) approach is that the plenoptic function $R(X, Y, Z, \varphi, \vartheta, \lambda)$ is described as the weighted sum of the $n_{\max }$ physical basis spectra $S_{n}(\lambda)$, which exists in the LED light source:

$$
R(X, Y, Z, \varphi, \vartheta, \lambda)=\sum_{n=1}^{n_{\max }} A_{n}(X, Y, Z, \varphi, \vartheta) \times S_{n}(\lambda)
$$

Equation 1 assumes that although the plenoptic function $R(X, Y, Z, \varphi, \vartheta, \lambda)$ varies spectrally as a function of the angular and spatial dimension, its underlying relative spectral distributions are constant. $R(X, Y, Z, \varphi, \vartheta, \lambda)$ can therefore be described by the weighted sum of this constant basis spectra. Thereby each basis spectrum requires a physical origin in the system. Then all spatial and angular variations are described by the amplitude distributions $A_{n}(X, Y, Z, \varphi, \vartheta)$, which change as a function of angular and spatial dimension.

However, $A_{n}(X, Y, Z, \varphi, \vartheta)$ cannot be measured directly because there is no optical filter, which generally measures only one basis spectrum. The $M_{n}(X, Y, Z, \varphi, \vartheta)$ obtained with the ILMD-based measurements depend on the effective spectral sensitivities $s_{\tau, \text { eff }, n}(\lambda)$ of the ILMD-system. The sensitivity $s_{\tau, \text { eff }, n}(\lambda)$ is the product of the transmission function of the actual optical filter $\tau_{n}(\lambda)$ used to perform the measurement, the optional transmission function of a neutral density filter $\tau_{\mathrm{ND}}(\lambda)$ and the spectral system sensitivity function $s_{\mathrm{Sys}}(\lambda)$. Thus, a measured ray file is defined as.

$$
M_{n}(X, Y, Z, \varphi, \vartheta)=\sum_{n=1}^{n_{\max }} A_{n}(X, Y, Z, \varphi, \vartheta) \times \int S_{n}(\lambda) \underbrace{\tau_{n}(\lambda) \tau_{\mathrm{ND}}(\lambda) s_{\text {Sys }}(\lambda)}_{s_{\tau, \mathrm{eff}, n}(\lambda)} d \lambda
$$

If it is assumed that besides the measured signal $M_{n}(X, Y, Z, \varphi, \vartheta)$ the relative basis spectra $S_{n}(\lambda)$ as well as all spectral transmissions and sensitivities and therefore $s_{\tau, \mathrm{eff}, n}(\lambda)$ are known, only the $n_{\max } A_{n}(X, Y, Z, \varphi, \vartheta)$ are unknown variables. If $m$ different optical filters are used, they produce $m$ different effective spectral sensitivities $s_{\tau, \text { eff, } m}(\lambda)$ and thus $m$ spectrally weighted measurement values $M_{n}(X, Y, Z, \varphi, \vartheta)$, which are given by

$$
\begin{aligned}
M_{1}(X, Y, Z, \varphi, \vartheta) & =\sum_{n=1}^{n_{\max }} A_{n}(X, Y, Z, \varphi, \vartheta) \times \int S_{n}(\lambda) s_{\tau, \mathrm{eff}, 1}(\lambda) d \lambda \\
M_{m}(X, Y, Z, \varphi, \vartheta) & =\sum_{n=1}^{n_{\max }} A_{n}(X, Y, Z, \varphi, \vartheta) \times \int S_{n}(\lambda) s_{\tau, \mathrm{eff}, m}(\lambda) d \lambda
\end{aligned}
$$


These equations are a system of linear equations, which has a unique solution if the number of different optical filter measurements equals the number of physical basis spectra $m=n_{\max }$. Equation 3 can be rewritten in its matrix form as

$$
\left[\begin{array}{c}
M_{1}(X, Y, Z, \varphi, \vartheta) \\
\vdots \\
M_{n_{\max }}(X, Y, Z, \varphi, \vartheta)
\end{array}\right]=\underbrace{\left[\begin{array}{ccc}
\int S_{1}(\lambda) s_{\tau, \mathrm{eff}, 1}(\lambda) d \lambda & \cdots & \int S_{n_{\max }}(\lambda) s_{\tau, \mathrm{eff}, 1}(\lambda) d \lambda \\
\vdots & \ddots & \vdots \\
\int S_{1}(\lambda) s_{\tau, \mathrm{eff}, S_{n_{\max }}}(\lambda) d \lambda & \cdots & \int S_{S_{n_{\max }}}(\lambda) s_{\tau, \mathrm{eff}, S_{n_{\max }}}(\lambda) d \lambda
\end{array}\right]}_{M_{S \tau}} \cdot\left[\begin{array}{c}
A_{1}(X, Y, Z, \varphi, \vartheta) \\
\vdots \\
A_{n_{\max }}(X, Y, Z, \varphi, \vartheta)
\end{array}\right]
$$

The matrix $M_{\mathrm{S \tau}}$ summarizes all available information about the effective spectral sensitivity of the measurement system and the physical basis spectra of the DUT. Eq. 4 therefore relates the measurement values $M_{n}(X, Y, Z, \varphi, \vartheta)$ to the unknown amplitudes $A_{n}(X, Y, Z, \varphi, \vartheta)$ which will be assigned to the light source model's $n_{\max }$ physical basis spectra. The required distributions $A_{n}(X, Y, Z, \varphi, \vartheta)$, can be reconstructed by inverting the matrix $M_{\mathrm{S \tau}}$. The inverted matrix $M_{\mathrm{S \tau}}^{-1}$ is called the spectral reconstruction matrix. The reconstructed distributions can be used to calculate the plenoptic function and thus to create the spectral ray file according to Eq. 1 .

It is important to note that these equations are only valid if absolute values are used. If absolute values are not available directly, they can be reconstructed by a comparison of the measured ray file and a spectral measurement within the goniometric setup. The details are described in [1]. Further, there are some limitations, which shall be mentioned. The most limiting assumption is the constant basis spectra assumption, which implies that PMBS does not cover nonlinear effects such as quenching caused by phosphor saturation due to a high radiant flux of the LED [20] or phosphor self absorption. Chromatic effects resulting from a primary optic cannot be modeled with the PMBS approach as well. Although it is common in near field photometry, it shall be mentioned that all interrelated measurements require the steady state condition with respect to electrical and thermal operation conditions.

\section{Workflow of PMBS}

Based on these relations, a general workflow for PMBS can be concluded. The workflow is visualized in Fig. 2 and serves as an outline for the next subsections. The process starts with the determination of the individual semiconductor and phosphor basis spectra using a spectral measurement. Subsequently, the goniophotometric measurement with $n_{\max }$ different suitable filters are performed. The spectral sensitivities of the measurement system and the basis spectra are combined to form the spectral reconstruction matrix $M_{\mathrm{S \tau}}^{-1}$. Subsequently, the ray files are used to solve the system of linear equations by applying the reconstruction equation Eq. 4 .

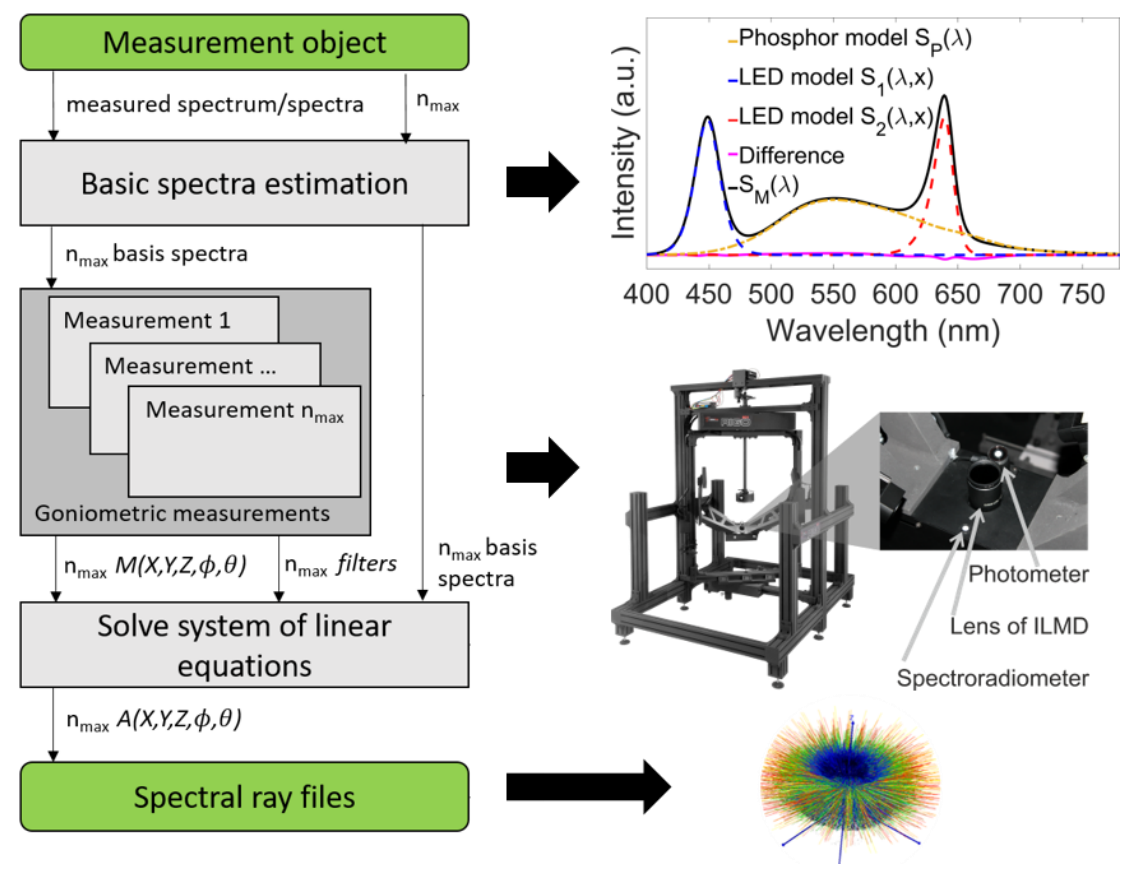

Figure 2: Workflow to apply PMBS (published under CC BY-SA 4.0 from [1]). 


\section{Basis spectra estimation}

Each LED basis spectrum can be described by a set of a model parameters $x_{n}=\left\{p_{1} \ldots p_{j}\right\}$, which parametrize a phenomenological model function such as a Gaussian distribution, a second order Lorentzian or an even more complex function. The number of parameters $j$ depend on the specific phenomenological model used. Examples are provided in $[21,22]$

In order to precisely estimate a set of basis spectra $S_{i}(\lambda)$ out of a measured LED spectrum $S_{\mathrm{M}}(\lambda)$, we assume that all basis spectra occur in the measurement and that at least the number of fundamental different basis spectra (different colors, phosphor) is known prior to the process. Based on this knowledge a set of model parameters $x=\left\{x_{1} \ldots x_{n_{\max }}\right\}$ can be estimated using an optimization algorithm, which minimizes the residual sum of squares between the sum of all basis spectra models and the measured spectrum.

$$
R S S=\min _{x \in \mathbb{R}} f(x) \text { with } f(x)=\sum_{\lambda}\left(S_{M}(\lambda)-\sum_{n}^{n_{\max }} S_{n}\left(\lambda, x_{n}\right)\right)^{2}
$$

In order to also include a phosphor spectrum into the modeling process, a more complex procedure is necessary. It basically includes the determination of the model spectrum, which has the least probability to be an LED spectrum. Than it applies a smoothing spline on the measured spectrum minus all LED spectra (excluding the determined spectrum). The process can be repeated until convergence is reached. More information and examples are provided in [1]

The basis spectra can be optimized by incorporating an additional LED attribute, which is the spatial separation of colored LEDs. Most often, the different LED colors lie next to each other. A green LED is green, a red LED is red and a white LED is white (or rather blue and yellow). The green, red and white LEDs do not overlap in the 3-dimensional spatial dimension. Although there might be a remote phosphor, which overlaps with all LEDs, the single colored chips are both spatially separated and highly localized in the three dimensional spatial dimension.

A set of single measurement images $M_{n}(X, Y)$ can be used to reconstruct the spectral images (or irradiances) $A_{n}(X, Y)$ of the individual basis spectra using the mathematical relations described in Eq. 4. The reconstructed $A_{n}(X, Y)$ can be used to quantify the "spatial separation" of the individual spectral sources and thus be used as additional merit or constraint for an additional optimization.

\section{Determination of optical filters}

In theory any arbitrary filter combination is sufficient for a spectral reconstruction with PMBS. An example is shown in Fig. 3. The RGBW LED system consists of four basis spectra and, therefore, PMBS requires four different ILMD-based filter measurements. According to Eq. 4, the four basis spectra can be reconstructed with, for instance, the filter combinations A-D. However, at a closer look no one would consider to choose filter combination B or C during the application of PMBS in this example because they do not provide an accurate measurement of the blue part of the spectrum. During the application of PMBS the ideal reconstruction Eq. 4 has to be extended to the real reconstruction Eq. 6 . This equation considers that the reconstructed $A_{n}(X, Y, Z, \varphi, \vartheta)$ differ by $\Delta A_{n}(X, Y, Z, \varphi, \vartheta)$ due to the deviations $\eta_{n}(X, Y, Z, \varphi, \vartheta)$ of the measurements $M_{n}(X, Y, Z, \varphi, \vartheta)$

$$
\left[\begin{array}{c}
M_{1}(X, Y, Z, \varphi, \vartheta)+\eta_{1}(X, Y, Z, \varphi, \vartheta) \\
\vdots \\
M_{n_{\max }}(X, Y, Z, \varphi, \vartheta)+\eta_{n_{\max }}(X, Y, Z, \varphi, \vartheta)
\end{array}\right]=M_{S \tau} \cdot\left[\begin{array}{c}
A_{1}(X, Y, Z, \varphi, \vartheta)+\Delta A_{1}(X, Y, Z, \varphi, \vartheta) \\
\vdots \\
A_{n_{\max }}(X, Y, Z, \varphi, \vartheta)+\Delta A_{n_{\max }}(X, Y, Z, \varphi, \vartheta)
\end{array}\right]
$$

The uncertainty $\eta_{n}(X, Y, Z, \varphi, \vartheta)$ from Eq. 6 has two different physical origins

$$
M_{n}(X, Y, Z, \varphi, \vartheta)+\eta_{n}(X, Y, Z, \varphi, \vartheta)=\left(M_{\mathrm{t}, n}(X, Y, Z, \varphi, \vartheta)+\eta_{\tau, n}(X, Y, Z, \varphi, \vartheta)\right)\left(1+\eta_{\mathrm{N}}\right) .
$$

The first factor consists of uncertainties $\eta_{\tau, n}(X, Y, Z, \varphi, \vartheta)$ regarding the effective spectral sensitivities $s_{\tau, \mathrm{eff}, n}(\lambda)$ and their interactions with the basis spectra. The uncertainty $\eta_{\tau, n}(X, Y, Z, \varphi, \vartheta)$ depends on the individual filter used in the measurement and is added to the true value $M_{\mathrm{t}, n}(X, Y, Z, \varphi, \vartheta)$. The second term $\eta_{\mathrm{N}}$ describes uncertainties, which apply for all filter measurements independent of the specific filter function $s_{\tau, \text { eff }, n}(\lambda)$ at each step of the goniophotometric measurements. Examples are noise from the sensor chip or the quantification errors caused by the ray file resolutions. 

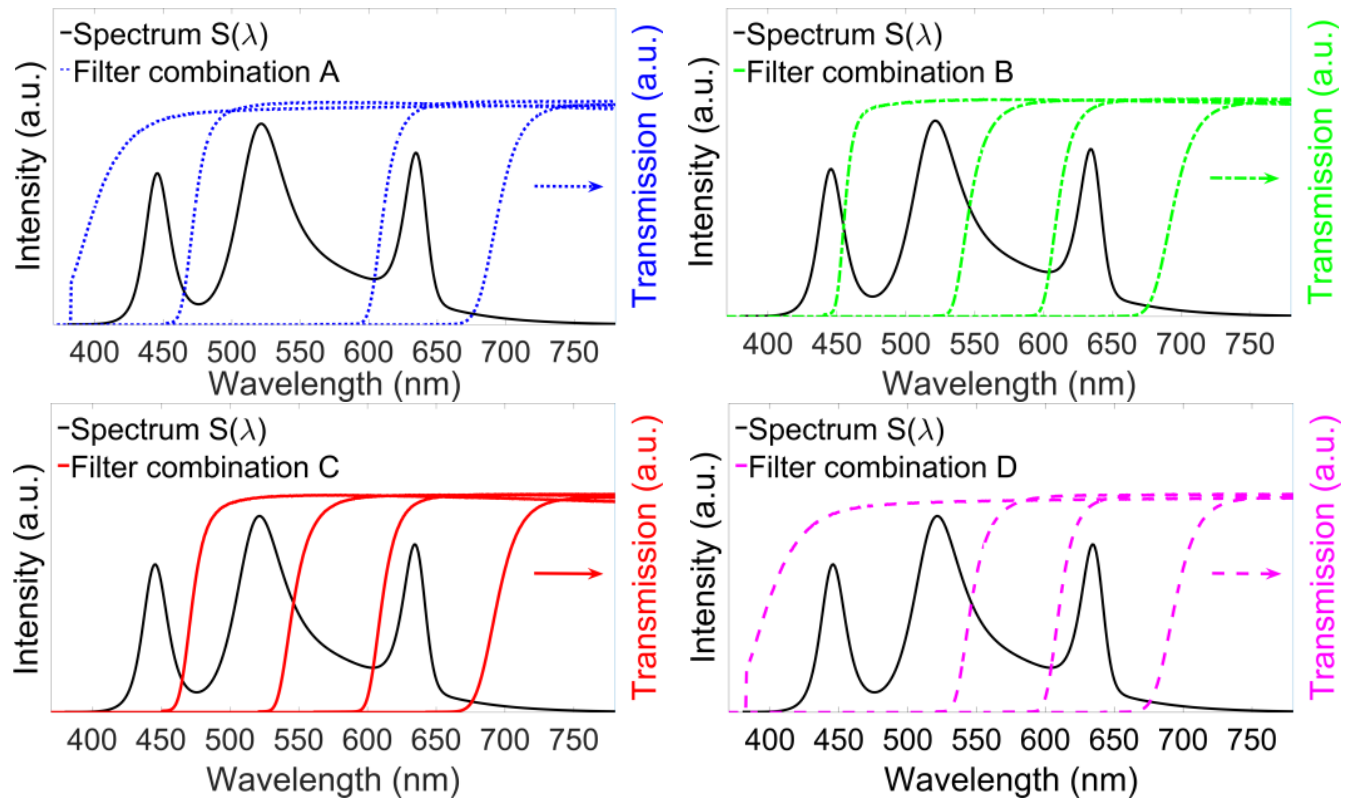

Figure 3: Different combinations of optical glass edge absorption filters to apply PMBS on a RGBW LED-system (published under CC BY-SA 4.0 from [1]).

A detailed estimation of the reconstruction performance of a filter combination needs to consider the individual filter based $\eta_{\tau, n}(X, Y, Z, \varphi, \vartheta)$ as well as its error propagation. Thus, the performance will differ for each filter combination and selecting a robust filter combination becomes an essential part of applying PMBS.

In order to determine a robust filter combination, a Monte Carlo simulation, which incorporates precise filter models, uncertainty distributions as well as the different filter combinations, can be carried out. Suitable models, uncertainty distributions and a modeling and evaluation procedure can be found in [23].

Figure 4 shows a comparison of a Monte Carlo simulation and a real reconstruction performance of 15 possible filter combinations for the RGBW system from Fig. 3. Altogether six glass absorption filters were considered. The y-axis shows the mean of the measured reconstruction performance as chromaticity coordinate distance $\Delta u^{\prime} v^{\prime}$. It bases on a comparison of the reconstructed spectral LED model to a angularly resolved spectral measurements in the goniometric setup. The $\mathrm{x}-$ axis shows the selection number/ranking of a specific filter combination. The lower the selection number, the better the simulated reconstruction performance.

The reconstruction performance can be divided into roughly three groups. The first group results in a $\Delta u^{\prime} v^{\prime} \approx 0.01$, the second group in a $\Delta u^{\prime} v^{\prime} \approx 0.025$ and the last group in a $\Delta u^{\prime} v>0.1$. The last group consists of filter combinations, which cannot reconstruct the spectra. Impossible chromaticity distance values such as $\Delta u^{\prime} v>0.6$ result from physically impossible chromaticity coordinates, which occur due to partly negative spectral distributions. The impossible area is marked in Fig. 4. The Monte Carlo based filter selection succeeds in separating these three groups. That way, the filter selection identifies a robust and suitable filter combination to apply PMBS.

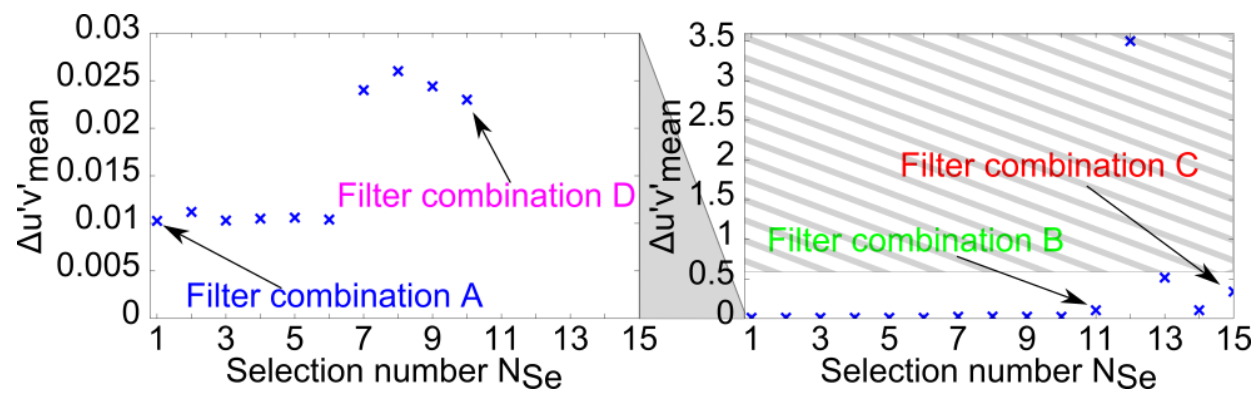

Figure 4: Validation of Monte Carlo based filter selection (published under CC BY-SA 4.0 from [1]). 


\section{VALIDATION}

This section is separated. into two parts. In the first part, a PMBS-based reconstruction of a phosphor converted white LED is compared to the Blue/Yellow approach, which is an approach that focus solely on this specific LED type. In the second part a spectral ray tracing simulation using a PMBS-based spectral ray file is compared to a colorimetric far field measurement of the same optical system. Details regarding the reconstructions are provided in [1].

\section{Comparison to the Blue/Yellow approach}

To quantitatively compare both methods, an LED with a strong angular color uniformity was chosen. Angularly resolved spectral measurements have been performed to assess the results. Figure 5 shows the LED as well as its color shadow on a photographic image. The angularly resolved spectral measurements are evaluated as chromaticity coordinates in the CIE $u^{\prime} v^{\prime}$ diagram and shown in Fig. 5 as well.
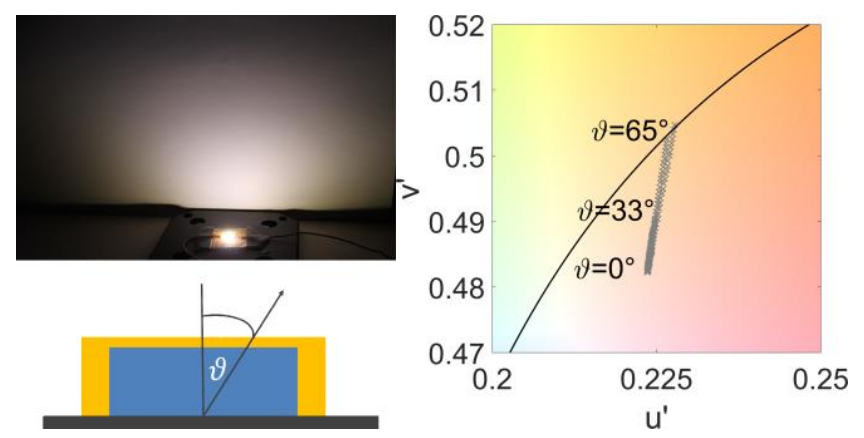

Figure 5: Tested white LED and measured angular color shift (published under CC BY-SA 4.0 from [1]).

Figure 6 shows the reconstructions of LED spectra as well as a comparison of the reconstructed chromaticity coordinates to the angularly resolved spectral measurements for both approaches. The comparison of reconstructed spectra to measured spectra shows that the deviations are smaller in the case of PMBS. The comparison of the reconstructed chromaticities to the measurements lead to the same conclusion. PMBS is not only able to describe the relative chromaticity shift more precisely, but also differs less in terms of the absolute chromaticity differences, which is $\Delta u^{\prime} v_{\text {mean }}^{\prime} \approx 0.002$ Note that the required measurement amount of both methods is identical in the case of a phosphor converted white LED.
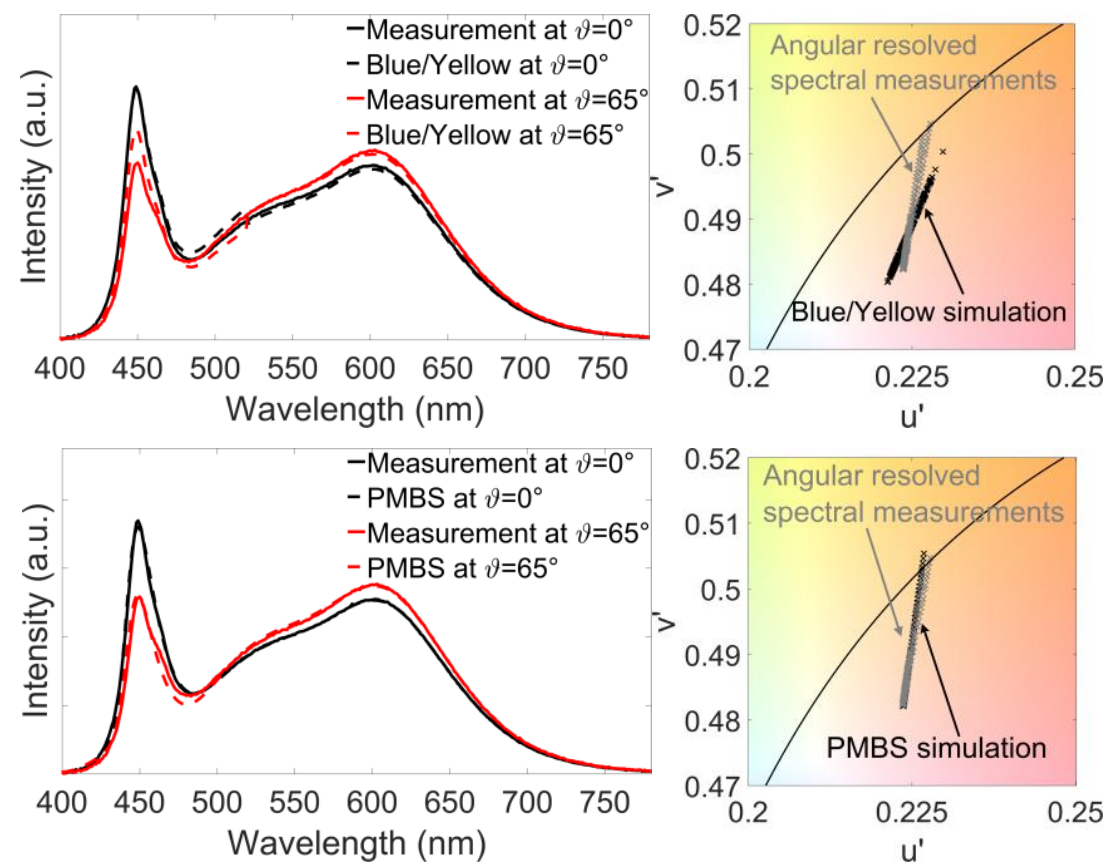

Figure 6: Comparison of the Blue/Yellow and PMBS approach (published under CC BY-SA 4.0 from [1]). 


\section{Spectral ray tracing}

To validate the suitability of the obtained spectral ray files, a far field measurement of a wavelength dependent optical system including the measurement object was compared to ray tracing simulations in the commercial ray tracer OptisWorks from OPTIS. The experimental setup containing the temperature controlled (TEC controller) LED source as well as the dispersion prism on a rotating stage is shown in Fig 7. The LED source uses three separated RGBW LEDs. In the experiment the configuration used is red (upper left), red-white, (middle), and white (bottom right), which can also be noticed in the photographic image of the experimental setup. The optical system consists of one dispersion prism because it shows a strong wavelength dependence and because the simple geometry minimizes deviations between the optical system and the CAD geometry, which can disturb the assessment of the spectral ray files.
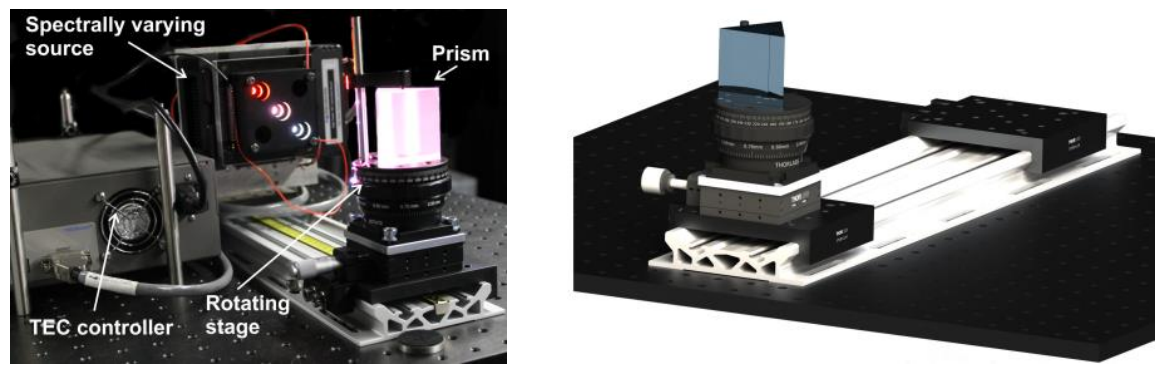

Figure 7: Experimental setup (left) and simulation setup (right) (published under CC BY-SA 4.0 from [1]).

The simulation setup is shown in Fig. 7 on the right hand side. In both simulation and measurement the prism was aligned with the rotating plate. As in the measurement, the photometric center of the intensity sensor in the simulation was defined at the center of the prism. The ray file editor from OptisWorks was used to assign the basis spectra to the reconstructed ray files. As the prism was located in the near field of the source, the influence of the prism is different for each LED source. Figure 8 shows a photographic image of the obtained distribution on the left hand side. It consists of a solely white part in the upper right, a red/white mixture in the middle and a solely red part in the lower left. Furthermore, both parts with a white LED show a chromatic shift from blue to red caused by the prism. The characteristic shape arises due to the placement of the source and the aperture of the prism.

A colorimetric intensity distribution of the refracted part of the light was goniometrically measured with an automotive goniometer and the colorimeter C3300 from LMT. The measurement result is shown in Figure 8 in the middle. The image on the right hand side shows the filtered result of the spectral ray tracing simulation in OptisWorks. The filtering was performed with the OPTIS XMP-filter in order to decrease the noise in the simulation. Furthermore, only the three major sequences, which represent $92 \%$ of the radiometric power reaching the sensor, contribute to the simulation result. Both images aim to visualize the results in true colors. Therefore, results from the measurement and the simulation provided in the CIE spectral tristimulus values were converted in RGB values. It has to be noted that the results remain false colors if watched on a printed page or a display. The comparison of Fig. 8 middle and right shows a very good qualitative agreement between the simulation and the measurement. Both the general shape and color of each region as well as the overlapping regions obtained with the spectral ray files fit the measurement. This can also be verified by the red spot in the upper left corner. It is caused by a reflection of the red LED within the prism and occurs in both measurement and simulation
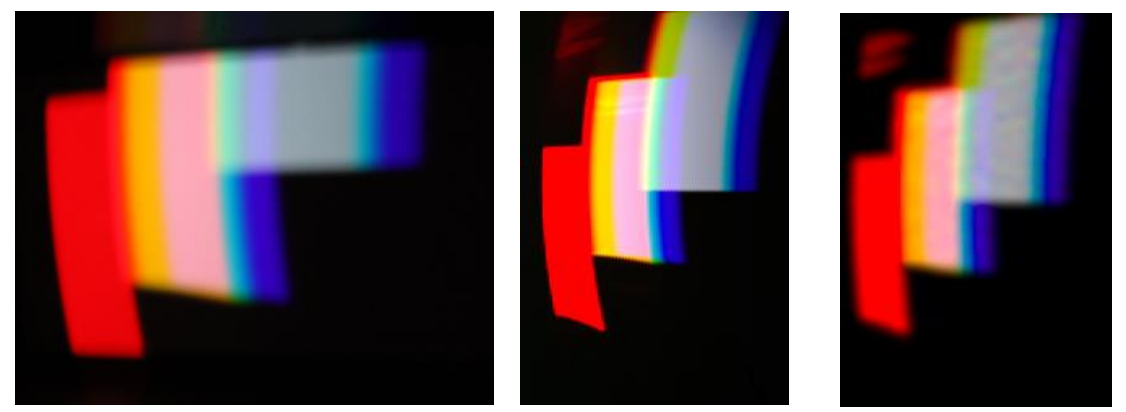

Figure 8: Qualitative comparison: Photographic Image (left), Colorimetric far field measurement (middle) and spectral ray tracing result (right) (published under CC BY-SA 4.0 from [1]). 


\section{SUMMARY AND CONCLUSION}

The aim of this contribution was to summarize the measurement and modeling technique PMBS proposed in [1], which can be used for obtaining the spectral near field of LEDs in order to improve the optical design of high quality LED based lighting systems. The main assumption of PMBS is that spectral variations in the near field of a LED system can be described as the weighted sum of all physically occurring spectral sources. This enables the possibility of interpreting a LED system as a set of spectral models, which can each be described individually, for instance as a ray file with one global spectral distribution. The main measurement effort in the form of conventional ILMD-based (imaging luminance measurement device) goniophotometric measurements with different optical filters is determined by the number of spectral sources and is therefore always minimized.

The basis spectra estimation requires at least one global spectral measurement and the number of different spectral sources in the system to obtain phenomenological LED basis spectra. If necessary, the basis spectra can be optimized by quantifying the "spatial separation". This figure of merit assumes that single colored chips are both spatially separated and highly localized in the three dimensional space. Furthermore, it has been shown that the optical filter selection influences the reconstruction performance. A suitable filter combination out of several possibilities can be estimated by a Monte Carlo simulation, which includes the basis spectra and models for optical filters as well as uncertainties. This procedure has been validated with an RGBW reconstruction.

Using the described implementation, PMBS was further used to reconstruct a conventional phosphor converted white LED. The reconstruction was compared to the industrial used Blue/Yellow approach. The reconstructions, which were obtained with PMBS, showed smaller deviations from validation measurements than those of the Blue/Yellow approach.

Finally, spectral ray files of a Red/White LED-system have been created. They were used within a commercial ray tracer to obtain a spectral far field distribution, created by a spectrally sensitive prism in the near field of the measurement object. The spectral simulations were compared to measured values of the same setup. Therefore, it can be concluded that the fast measurement method PMBS is suitable for obtaining spectral ray files, which can be used to improve the optical design of high quality LED based lighting systems.

\section{ACKNOWLEDGEMENTS}

This work was supported by the Federal Ministry of Education and Research in the Program Photonics Research Germany (Funder Id: 10.13039/501100002347, contract number 13N13396).

\section{REFERENCES}

[1] Rotscholl, I., [Spectral Near Field Data of LED Systems for Optical Simulation], KIT Scientific Publishing, (2018).

[2] Taguchi, T., Uchida Y., and Kobashi, K. "Efficient white LED lighting and its application to medical fields," physica status solidi (a) 201(12), 2730-2735 (2004).

[3] Kobashi, K., and Taguchi, T., "Warm white LEDs lighting over Ra=95 and its applications," Proc. SPIE 6486, (2007).

[4] Morrow, R. C., "LED Lighting in Horticulture," HortScience 43(7), 1947-1950 (2008).

[5] Bhardwaj, J., Peddada, R., and Spinger, B., "Advances in LEDs for automotive applications," Proc. SPIE 9768, (2016).

[6] Almeida, A. D., Santos, B., Paolo, B., and Quicheron, M., "Solid state lighting review - Potential and challenges in Europe," Renewable and Sustainable Energy Reviews 34(Supplement C), 30 - 48 (2014).

[7] Wendel, S., [Freiform-Optiken im Nahfeld von LEDs], KIT Scientific Publishing, (2014).

[8] Wang, K., Chen, F., Liu, Z., Luo, X., and Liu, S., "Design of compact freeform lens for application specific lightemitting diode packaging," Optics Express 18(2), 413-425 (2010).

[9] Schubert, E. F., and Kim, J. K., "Solid-state light sources getting smart," Science 308(5726), 1274-1278 (2005).

[10] Katona, T. M., Pattison, P. M., and Paolini S., "Status of Solid State Lighting Product Development and Future

Trends for General Illumination," Annual Review of Chemical and Biomolecular Engineering 7(1), 263-281 (2016).

[11] Boyce P. R., [Human Factors in Lighting], CRC Press (2014).

[12] Rykowsky R., "Spectral ray tracing from near field goniophotometer measurements," Light \& Engineering 19(1), 23-29 (2011). 
[13] Hansen D., "Messung und Simulation polychromatischer Strahldaten," Automobiltechnische Zeitschrift 114(11), 869-873 (2012).

[14] Hansen, D., Paul, S., and Schumacher V., "LED Measurement to Obtain Polychromatic Raydata and their Value for Simulations," LED Professional Symposium, (2012).

[15] Jacobs, V. A., Audenaert, J., Bleumers, J., Durinck, G., Rombauts, P., and Hanselaer, P., "Rayfiles including spectral and colorimetric information," Optics Express 23(7), A361-A370 (2015).

[16] OSRAM Opto Semiconductors GmbH, "Application Note: Importing Rayfiles of LED from OSRAM Opto Semiconductors," (2013).

[17] Lee, T.-X., Lu, T.-L., and Chen, B.-S., "Combining near-field hyperspectral imaging and far-field spectral-angular distribution to develop mid-field white LED optical models with spatial color deviation," Optics Express 24(14), A1094-A1106 (2016).

[18] Sun, C.-C., Lee, T.-X., Ma, S.-H., Lee, Y.-L., and Huang, S.-M., "Precise optical modeling for LED lighting verified by cross correlation in the midfield region," Optics Letters 31(14), 2193-2195 (2006).

[19] Adelson, E. H., and Bergen, J. R., "The Plenoptic Function and the Elements of Early Vision," in Computational Models of Visual Processing, 3-20 (1991).

[20] Setlur, A. A., "Phosphors for LED-based solid-state lighting," The Electrochemical Society Interface, 16(4), 32-36 (2009).

[21] Reifegerste, F., and Lienig, J., "Modelling of the Temperature and Current Dependence of LED Spectra," Journal of Light \& Visual Environment 32(3), 288-294 (2008).

[22] Khanh, T. Q., Bodrogi, P., and Vinh, T. Q., [Color Quality of Semiconductor and Conventional Light Sources], John Wiley \& Sons, (2017).

[23] Rotscholl, I., Trampert, K., Krüger, U., Perner M., Schmidt, F., and Neumann, C., "Determination of tailored filter sets to create rayfiles including spatial and angular resolved spectral information," Optics Express, 23(23) 2954329554 (2015). 